\title{
ON AUTOMORPHISMS OF A KÄHLERIAN STRUCTURE
}

\author{
SHOSHICHI KOBAYASHI and KATSUMI NOMIZU
}

Is every isometry, or more generally, every affine transformation of a Kählerian manifold a complex analytic transformation? The answer is certainly negative in the case of a complex Euclidean space. This question has been recently studied by Lichnerowicz [8] and Schouten-Yano [11] from the infinitesimal point of view; they have found some conditions in order that every infinitesimal motion of a Kählerian manifold preserve the complex structure. (As a matter of fact, [11] has dealt with the case of a pseudo-Kählerian manifold, which does not differ essentially from a Kählerian manifold as far as the question at hand is concerned.)

In the present paper, we generalize their results by a different approach. In order to explain our main idea, we shall first give a few definitions ( 1 and 2) and state our main results (3). The proofs are given in the subsequent sections.

\section{Kählerian structures}

Let $M$ be a complex analytic manifold of complex dimension $n$. Its complex structure is defined by a real analytic tensor field $I$ of type $(1,1)$ with $I^{2}=-\mathbf{1}^{1)}$ on the underlying $2 n$-dimensional real analytic manifold which satisfies the condition of integrability $I[X, Y]-[I X, Y]-[X, I Y]-I[I X, I Y]=0$ for all real vector fields $X$ and $Y$ (for example, [1]). A differentiable transformation $f$ of $M$ is said to preserve the complex structure $I$ if $\delta f \circ I=I \circ \delta f$, where $\delta f$ denotes the differential of $f$. This is equivalent to saying that $f$ is a complex analytic transformation. If $\delta f \circ I=-I \circ \delta f$, we say that $f$ maps $I$ into the conjugate complex structure $-I ; f$ is then a conjugate analytic transformation.

A real analytic Riemannian metric $g$ on a complex analytic manifold $M$ is called Kählerian if it is hermitian, that is, $g(I X, I Y)=g(X, Y)$ for all real vector fields $X$ and $Y$, and if $I$ is a parallel tensor field with respect to the

Received July 23, 1956.

1) Throughout the present note, 1 denotes the identity transformation. 
Riemannian connection arising from g. Likewise, given a real analytic manifold $M$ with Riemannian metric $g$, we shall say that a complex structure $I$ on $M$ is Kählerian if $g$ is Kählerian with respect to $I$ in the above sense. Such a pair $(I, g)$ defines a Kählerian structure on $M$. By an isometry (resp. affine transformation) of a Kählerian manifold $M$, we understand of course an isometry (resp. affine transformation) of the underlying Riemannian manifold. By an automorphism of $M$, we mean an isometry which preserves the complex structure.

A Kählerian manifold $M$ (with complex structure $I$ and Riemannian metric $g$ ) will be called non-degenerate if the restricted homogeneous holonomy group $\sigma_{x}$ of the underlying Riemannian manifold contains the endomorphism $I_{x}$ of the tangent space $T_{x}$ at $x \in M$, where $x$ is an arbitrary reference point for the holonomy group and $I_{x}$ is the value at $x$ of the tensor field $I$. Note that the condition $I_{x} \in \sigma_{x}$ is independent of the choice of a reference point $x$. For any point $y$, let $\tau$ denote the parallel displacement: $T_{x} \rightarrow T_{y}$ along an arbitrary curve from $x$ to $y$. Since $I$ is a parallel tensor field, we have $I_{y}=\tau \cdot I_{x} \cdot \tau^{-1}$. On the other hand, we have $\sigma_{y}=\tau \cdot \sigma_{x} \cdot \tau^{-1}$.

Finally, we shall say that a Riemannian manifold $M$ of dimension $>1$ is irreducible if the restricted homogeneous holonomy group is irreducible, that is, if it does not admit any non-trivial invariant subspace as a group of linear transformations on the (real) tangent vector space.

\section{Complex and quaternionian structures on a real vector space}

In this section, we shall indicate an intrinsic way of defining real representations of $G L(n, C), U(n), S U(n)$ or $G L(n, Q), S p(l)$, etc. such as described in C. Chevalley: Theory of Lie Groups I, Chapter I. The exposition is quite elementary but important for our purpose.

A complex structure $I$ on a real $m$-dimensional vector space $T$ is, by definition, an endomorphism of $T$ such that $I^{2}=-\mathbf{1}$. It allows us to define the set $T$ as an $n$-dimensional vector space over the field of complex numbers $C$, where $m=2 n$. More precisely, we define

$$
(a+b i) X=a X+b I X
$$

for $a, b \in R$ (field of real numbers) and $X \in T$. We denote by $T^{*}$ the vector space over $C$ thus obtained.

If $g$ is a positive definite inner product on $T$ such that $g(I X, I Y)=g(X, Y)$ 
for all $X, Y \in T$, then we may define a positive definite hermitian inner product $g^{*}$ on $T^{*}$ by

$$
g^{*}\left(X, Y^{\prime}\right)=g(X, Y)-i g(I X, Y)
$$

for $X, Y \in T^{*}$, where $X$ and $Y$ are considered as elements of $T$ on the right hand side of the above equation.

If $\tau$ is an endomorphism of $T$ which commutes with $I$, then it may be considered as an endomorphism $\tau^{*}$ of $T^{*}$, as is clear from $\tau(i X)=\tau(I X)=I(\tau X)$ $=i \tau X$. If furthermore $\tau$ leaves $g$ invariant, then $\tau^{*}$ leaves $g^{*}$ invariant.

We shall say that a group of linear transformations of a real vector space $T$ of dimension $m=2 n$ is contained in a real representation of $U(n)$ if $T$ admits a complex structure $I$ which commutes with every $\tau \in G$ and a positive definite inner product $g$ which is invariant by $I$ and every $\tau \in G$. In this case, $G$ is isomorphic with the subgroup $G^{*}=\left\{\tau^{*} ; \tau \in G\right\}$ (in the above notation) of the unitary group on $T^{*}$ with respect to $g^{*}$. If furthermore det $\tau^{*}=1$ for every $\tau \in G$, then we say that $G$ is contained in a real representation of $S U(n)$.

By a quaternionian structure on a complex vector space $T^{*}$ of dimension $n$, we shall mean a conjugate linear transformation $J$ of $V$ with $J^{2}=-1$, that is, a 1-1 map of $T^{*}$ onto itself such that

$$
J(X+Y)=J X+J Y, \quad J(a X)=\bar{a} J X \text { and } J^{2} X=-X
$$

for all $X, Y \in T^{*}$ and $a \in C$, where $\bar{a}$ denotes the complex conjugate of $a$. It allows us to consider $T^{*}$ as a vector space $\tilde{T}$ over the field of quaternions $Q$ in the following fashion. We represent every quaternion $q$ in the form $q=a+b j$ ( $j$ being an element of $Q$ such that $j^{2}=-1$ and $i j=-j i$ ) and define the scalar multiplication by

$$
q \cdot X=a X+b J X
$$

for all $X \in T^{*}$. As a vector space over $Q, \widetilde{T}$ is of dimension $l$ where $n=2 l$.

If $g^{*}$ is a positive definite hermitian inner product on $T^{*}$ such that $g^{*}(J X, J Y)=g^{*}(Y, X)\left(=\overline{g^{*}(X, Y)}\right)$, then we can define a positive definite symplectic inner product $\widetilde{g}$ in the vector space $\widetilde{T}$ over $Q$ in the following fashion:

$$
\widetilde{g}\left(X, Y^{*}\right)=g^{*}(X, Y)+g^{*}(X, J Y) j
$$

for all $X, Y \in \widetilde{T}$. Namely, $\widetilde{g}$ is $Q$-valued and satisfies the following conditions: 
1) $\widetilde{g}(Y, X)$ is the symplectic conjugate of $\widetilde{g}(X, Y)$;

2) $\widetilde{g}\left(X+X^{\prime}, Y\right)=\widetilde{g}(X, Y)+\widetilde{g}\left(X^{\prime}, Y\right)$;

3) $g(X, X) \geqslant 0$ for every $X$ and it is 0 if and only if $X=0$.

If $\tau^{*}$ is an endomorphism of $T^{*}$ over $C$ which commutes with $J$, then it may be regarded as an endomorphism $\tilde{\tau}$ of $\widetilde{T}$ over $Q$. If furthermore $\tau^{*}$ leaves $g^{*}$ invariant, then $\tilde{\tau}$ leaves $\widetilde{g}$ invariant.

We say that a group $G^{*}$ of linear transformations of a complex vector space $T^{*}$ of dimension $n=2 l$ is contained in a complex representation of $S_{p}(l)$ if $T^{*}$ admits a quaternionian structure $J$ and a positive definite hermitian inner product $g^{*}$ such that

$$
g^{*}(J X, J Y)=g^{*}(Y, X), \quad \tau^{*} \cdot J=J \cdot \tau^{*} \quad \text { and } \quad g^{*}\left(\tau^{*} X, \tau^{*} Y\right)=g^{*}(X, Y)
$$

for all $X, Y \in T^{*}$ and $\tau^{*} \in G^{*}$. In this case, $G^{*}$ is isomorphic with a subgroup of $S_{p}(l)$ on the vector space $\tilde{T}$ over $Q$ with respect to the symplectic inner product $\widetilde{g}$.

Finally, we define a quaternionian structure on a real vector space $T$. It is a pair of endomorphisms $I$ and $J$ of $T$ such that $I^{2}=J^{2}=-\mathbf{1}$ and $I J=-J I$. Such a structure makes it possible to regard $T$ as a vector space $\tilde{T}$ over $Q$, the scalar multiplication being defined by

$$
(a+b i+c j+d k) X=a X+b I X+c J X+d(1 J) X
$$

for $a, b, c, d \in R$ and $X \in T$. Another way of seeing this is to consider, first, $T$ with a complex structure $I$ as a vector space $T^{*}$ over $C$ and then consider the given endomorphism $J$ as a quaternionian structure on $T^{*}$, which is obviously possible.

This being said, we are able to use the following expression. We say that a group of linear transformations $G$ on a real $m$-dimensional vector space $T$ is contained in a real representation of $S_{p}(l)$, with $m=4 l$, if $T$ admits a quaternionian structure $(I, J)$ and a positive definite inner product $g$ which are both invariant by $I, J$ and every element of $G$. It is now easy to see that, in this case, $G$ is isomorphic with a subgroup of $S_{p}(l)$ on the $l$-dimensional vector space $\widetilde{T}$ over $Q$ with a suitable symplectic inner product.

By using the fact that $S_{p}(l)$ is connected, we can prove that if $G$ is contained in a real representation of $S_{p}(l)$, then it is contained in a real representation of $S U(n)$, where $m=2 n$ and $n=2 l$. We omit the detail of the proof. 


\section{.3. Main results}

THEOREM 1. Every simply connected and complete Kählerian manifold $M$ is a direct product $M_{0} \times M_{1} \times \ldots \times M_{k}$, where $M_{0}$ is a complex Euclidean space of dimension $\geqslant 0$ and $M_{1}, \ldots, M_{k}$ are irreducible Kählerian manifolds. If $M$ is non-degenerate, $M_{0}$ does not appear and $M_{1}, \ldots, M_{k}$ are all non-degenerate.

THEOREM 2.' Let $M$ be an irreducible Kählerian manifold whose restricted homogeneous holonomy group is not contained in a real representation of $S_{p}(l)$, where $\operatorname{dim} M=4 l$. Then every affine transformation of $M$ preserves the complex structure I or maps I into the conjugate complex structure. The largest connected group of affine transformations $A^{0}(M)$ preserves the complex structure.

Theorem 3. If $M$ is a complete non-degenerate Kählerian manifold, then the largest connected group of affine transformations $A^{0}(M)$ consists of automorphisms.

If $M$ is a pseudo-Kählerian manifold, we can still define the notion of nondegeneracy. If we replace "Kählerian" by "pseudo-Kählerian" and "complex structure" by "almost complex structure" respectively, then all the results stated above remain true.

It is of some interest to compare our problem with the following: is every affine transformation of a Riemannian manifold an isometry? This question has been settled by Hano and one of the authors as follows. Every simply connected and complete Riemannian manifold $M$ is a direct product of a Euclidean space $M_{0}$ and irreducible Riemannian manifolds $M_{1}, \ldots, M_{k}$ (the so-called de Rham decomposition) [10]. The largest connected group of affine transformations $A^{0}(M)$ is naturally isomorphic with $A^{0}\left(M_{0}\right) \times A^{0}\left(M_{1}\right) \times \ldots \times A^{0}\left(M_{k}\right)$ [2]. On the other hand, every affine transformation of a complete irreducible Riemannian manifold is an isometry [5]. It follows that, if $M$ is a complete Riemannian manifold whose restricted homogeneous holonomy group does not leave any non-zero vector invariant, then $A^{0}(M)$ consists of isometries.

Our Theorem 1 corresponds to the de Rham decomposition of a Riemannian manifold. By using the above result of Hano, our problem is reduced to the case of an irreducible Kählerian manifold, to which Theorem 2 is an answer. Here we do not need the condition of completeness but require an assumption

2) A similar result has been obtained also by $\mathrm{M}$. Obata. 
on the holonomy group. Now, what is a condition which assures that every component of the de Rham decomposition of a given Kählerian manifold satisfies the assumption of Theorem 2 ? The non-degeneracy is such a condition. The following theorem shows the relationship of this notion to the known facts on Ricci curvature, thus giving a heuristic interpretation of the results of Lichnerowicz [7], [8].

Theorem 4. Let $M$ be a Kählerian manifold of complex dimension $n$.

1) If $M$ is irreducible and the Ricci curvature is not zero, then $M$ is nondegenerate.

2) If $M$ is non-degenerate and $n$ is not divisible by 4, then the Riccicurvature of $M$ is not zero.

3) If the Ricci curvature of $M$ is non-singular at some point of $M$, then $M$ is non-degenerate.

Finally we add

Corollary. Let $M$ be a $2 n$-dimensional simply connected real analytic Riemannian manifold which is irreducible. Then the following three cases are possible:

1) If the restricted homogeneous holonomy group $\sigma$ is not contained in a real representation of $U(n)$, there exists no Kählerian structure at all on $M$.

2) If $\sigma$ is contained in a real representation of $U(n)$ but not of $S_{p}(l), n=2 l$, then there exist exactly two Kählerian structures on $M$ which are mutually conjugate.

3) If $\sigma$ is contained in a real representation of $S_{p}(l), n=2 l$, then there exist continuously may distinct Kählerian structures on $M$.

\section{Proof of Theorem 1}

The underlying Riemannian manifold of $M$ admits the de Rham decomposition $M=M_{0} \times M_{1} \times \ldots \times M_{k}$. It is not difficult (see [3]) to see that every component $M_{i}$ has a Kählerian structure induced from that of $M$ and that $M$ is the direct product of $M_{0}, M_{1}, \ldots, M_{k}$ as Kählerian manifolds. The homogeneous holonomy group $\sigma(M)$ of $M$ is decomposed into the direct product of the homogeneous holonomy groups $\sigma\left(M_{i}\right)$ of $M_{i}, i=0,1, \ldots, k$, where $\sigma\left(M_{0}\right)$ consits of the identity only. It follows that if $M$ is non-degenerate, the Euclidean 
part $M_{0}$ does not exist and the irreducible components $M_{i}, i=1,2, \ldots, k$, are all non-degenerate.

\section{Proof of Theorem 2}

Let $f$ be an affine transformation of $M$ and $\delta f$ its differential. Then $I^{f}=\delta f^{-1} \cdot I \cdot \delta f$ is a tensor field of type $(1,1)$ which clearly satisfies the condition $I^{f} \cdot I^{f}=-1$. We show that it is a parallel tensor field. Let $c$ be an arbitrary curve from $x$ to $y$ and let $\tau$ be the linear mapping of $T_{x}$ onto $T_{y}$ defined by parallel displacement along $c$. Let $c^{*}$ be the image curve of $c$ by $f$ and let $\tau^{*}$ be the linear mapping of $T_{f(x)}$ onto $T_{f(y)}$ defined by parallel displacement along $c^{*}$. Since $f$ is an affine transformation, we have $\delta f \cdot \tau=\tau^{*} \cdot \delta f$ on $T_{x}$ [9]. On the other hand, we have $I_{f(y)} \cdot \tau^{*}=\tau^{*} \cdot I_{f(x)}$ since $I$ is a parallel tensor field. Therefore we get

$$
\begin{aligned}
I_{y}^{f} \cdot \tau & =\delta f^{-1} \cdot I_{f(y)} \cdot \delta f \cdot \tau=\delta f^{-1} \cdot I_{f(y)} \cdot \tau^{*} \cdot \delta f \\
& =\delta f^{-1} \cdot \tau^{*} \cdot I_{f(x)} \cdot \delta f=\tau \cdot \delta f^{-1} \cdot I_{f(x)} \cdot \delta f=\tau \cdot I_{x}^{f},
\end{aligned}
$$

which proves our assertion. In particular, $I_{x}^{f}$ commutes with every element of $\sigma_{x}$.

Now let $A$ be the algebra (over the field of real numbers $R$ ) formed by all endomorphisms of $T_{x}$ which commute with every element of $\sigma_{x}$. Since $\sigma_{x}$ is irreducible, every non-zero element of $A$ has an inverse, that is, $A$ is a division algebra. By a well known theorem in algebra, $A$ is isomorphic either with the field of real numbers $R$, or the field of complex numbers $C$, or else the field of quaternions $Q$. Since $A$ contains an element $I_{x}$ with $I_{x}^{2}=-1$, it cannot be isomorphic with $R$. If $A$ were isomorphic with $Q$, it would follow that $\sigma_{x}$ is contained in a real representation of $S_{p}(l)$, with $n=2 l$; indeed, again by the irreducibility of $\sigma_{x}$, we see that the inner product $g_{x}$ of $T_{x}$ induced from the Kählerian metric of $M$ is invariant by the elements $I$ and $J$ of $A$ which correspond to the units $i$ and $j$ of $Q$. Hence $A$ is isomorphic with $C$. The only complex numbers whose square are -1 are $i$ and $-i$. Since $I_{x}^{f}$ is in $A$ and $I_{x}^{f} \cdot I_{x}^{f}=-1$, we have either $I_{x}^{f}=I_{x}$ or $I_{x}^{f}=-I_{x}$. Since $I$ and $I^{f}$ are parallel tensor fields, we have $I^{f}=I$ or $I^{f}=-I$. This concludes the proof of the first part of Theorem 2 .

In order to prove the second part, let $f$ be an element of $A^{0}(M)$. We take a continuous 1-parameter family $f_{t}$ of affine transformations such that $f_{0}=$ identi- 
ty transformation and $f_{1}=f$. If we form $I^{t}=\delta f_{t}^{-1} \cdot I \cdot \delta f_{t}$, then we have $I_{x}^{t}=I_{x}$ or $-I_{x}$ from what we have seen. Since $I_{x}^{t}$ is a continuous 1-parameter family of endomorphisms of $T_{x}$ such that $I_{x}^{t}=I_{x}$ for $t=0, I_{x}^{t}$ must coincide with $I_{x}$ for every $t$. In particular, we have $I_{x}^{f}=I_{x}$, that is, $I^{f}=I$. This proves that $f$ preserves the complex structure $I$.

\section{Proof of Theorem 3}

Let $\tilde{M}$ be the universal covering manifold of $M$ provided with a naturally induced Kählerian structure. It is easy to see that $\tilde{M}$ is also complete and nondegenerate. By the argument in [2], it is sufficient to prove Theorem 3 for $\tilde{M}$. By Theorem 1, $\tilde{M}=M_{1} \times \ldots \times M_{k}$ where each $M_{i}$ is irreducible and nondegenerate. The homogeneous holonomy group $\sigma\left(M_{i}\right)$ is not contained in a real representation of $S_{p}(l), n=2 l$. In fact, we show that the division algebra $A$ considered in the proof of Theorem 1 cannot be isomorphic with $Q$. If it were so, the elements $I, J$ and $K$ of $A$ corresponding to $i, j$ and $k \in Q$ must commute with the element $I_{0}, I_{0}^{2}=-\mathbf{1}$, of $A$ determined by the given complex structure of $M_{i}$, which is contained in $A$ since $M_{i}$ is non-degenerate. This is a contra-

diction. Hence Theorem 2 shows that $A^{0}\left(M_{i}\right)$ preserves the complex structure of $M_{i}$. Since $A^{0}(\tilde{M})$ is the direct product of $A^{0}\left(M_{i}\right), i=1,2, \ldots, k$, we see that $A^{0}(\tilde{M})$ preserves the complex structure of $\tilde{M}$. On the other hand, we already know ([2] and [5]) that $A^{0}(\tilde{M})$ consists of isometries. Hence $A^{0}(\tilde{M})$ consists of automorphisms of $\tilde{M}$.

\section{Proof of Theorem 4}

1) The complex structure $I_{x}$ of the tangent space $T_{x}$ is invariant by the restricted homogeneous holonomy group $\sigma_{x}$ operating on $T_{x}$, we may consider $\sigma_{x}$ as a subgroup of $U(n)$ as indicated in 2 . Since the Ricci curvature is not zero, $\sigma_{x}$ is not a subgroup of $S U(n)$ ([7], see also [4] and [6]) which means that $\sigma_{x}$ has a non-discrete center. $\sigma_{x}$ being irreducible, the center must be of dimension 1 by Schur's lemma. Hence $\sigma_{x}$ contains the 1-parameter subgroup $\left\{e^{2 \pi i r} \cdot 1 ; r\right.$ reals $\}$ of $U(n)$, in particular, the transformation $i$. In real representation, this means that $\sigma_{x}$ contains the endomorphism $I_{x}$, that is, $M$ is non-degenerate.

2) If we consider $\sigma_{x}$ as a subgroup of $U(n)$, then $\sigma_{x}$ contains the transformation $i 1$ whose determinant, the $n$-th power of $i$, is not equal to 1 since $n$ is 
not divisible by 4 . Hence $\sigma_{x}$ is, not a subgroup of $S U(n)$ and the Ricci curvature is not zero.

3) Let $x$ be a point of $M$ where the Ricci curvature is non-singular. Let $U$ be a properly chosen neighborhood of $x$ which is isometric to the direct product $U_{0} \times U_{1} \times \ldots \times U_{k}$, where $U_{0}$ is locally a complex Euclidean space and $U_{1}, \ldots, U_{k}$ are irreducible Kählerian manifolds (the argument is similar to that in the proof of Theorem 1). We may consider $U_{0}, U_{1}, \ldots, U_{r}$ as submanifolds of $U$ passing through $x$. Then the Ricci curvature of $U$ at $x$ is the direct sum of the Ricci curvatures of $U_{0}, U_{1}, \ldots, U_{k}$. Therefore the Ricci curvature of each $U_{i}$ is non-singular at $x$. It follows that there does not exist $U_{0}$ and that $U_{1}, \ldots, U_{k}$ are non-degenerate by 1$)$ of Theorem 4. Since $\sigma_{x}(M)$ contains $\sigma_{x}(U)=\sigma_{x}\left(U_{1}\right) \times \ldots \times \sigma_{x}\left(U_{k}\right)$, we see that $\sigma_{x}(M)$ contains the endomorphism $I_{x}$.

\section{Proof of Corollary}

At any reference point $x$, we consider the division algebra $A$ formed by all endomorphisms of $T_{x}$ commuting with every element of $\sigma_{x}$. If $A$ is isomorphic with $R$, then there is no element $I \in A$ with $I^{2}=-\mathbf{1}$; there is no Kählerian structure on $M$. If $A$ is isomorphic with $C$, then let $I_{x}$ be an element of $A$ with $I_{x}^{2}=\mathbf{- 1}$. By parallel displacement of $I_{x}$, we get a parallel tensor field $I$ of type $(1,1)$ such that $I^{2}=-1$ and $g(I X, I Y)=g(X, Y)$. It is known that an almost complex structure which is parallel with respect to a Riemannian connection (or, more generally, an affine connection without torsion) is integrable [1]. Hence $I$ is a Kählerian structure. It is clear that $I$ and its complex conjugate structure $-I$ are the only Kählerian structures on $M$.

If $A$ is isomorphic with $Q$, then it contains continuously many elements $S$ with $S^{2}=-1$. Namely, if $I, J$ and $K$ are the elements of $A$ which correspond to $i, j$ and $k$ of $Q$ respectively, then we may take $S=b I+c J+d K$, where $b, c$ and $d$ are real numbers such that $b^{2}+c^{2}+d^{2}=1$. For any such element $S$ of $A$, we get a Kählerian structure on $M$ by the same argument as before. Hence $M$ has continuously many distinct Kählerian structures.

\section{Remarks}

In the case of a compact Kählerian manifold $M$, the largest connected group of affine transformations $A^{0}(M)$ consists of isometries (theorem of Yano, 
which has been generalized in [7]) and preserves the complex structure of $M$, as is remarked in [8]. Indeed, the form $F$ associated to the Kählerian structure of $M: F(X, Y)=g(I X, Y)$ is harmonic and invariant by every 1-parameter group of isometries. It follows that $I$ is also invariant by the 1-parameter group.

The above statement is no longer true for the total group $A(M)$ of affine transformations. For example, in a complex projective space $P_{n}$ with usual Fubini-Study metric, the transformation defined by $\left(z^{0}, z^{1}, \ldots, z^{n}\right) \rightarrow\left(\bar{z}^{0}, \bar{z}^{1}\right.$, $\left.\ldots, \bar{z}^{n}\right)$ in terms of homogeneous coordinates $z^{0}, \ldots, z^{n}$ is isometric but not complex analytic.

\section{BIBLIOGRAPHY}

[1] Eckmann, B., Sur les structures complexes et presque complexes, Colloque de Géométrie Différentielle, Strasbourg (1953), 151-159.

[2] Hano, J., On affine transformations of a Riemannian manifold, Nagoya Math. J. 9 (1955), 99-109.

[3] Hano, J. and Matsushima, Y., Some studies on Kählerian homogeneous spaces, this journal.

[4] Iwamoto, H., On the structure of Riemannian space whose holonomy groups fix a null-system, Tohoku Math. J. 2nd ser. 1 (1950), 109-125.

[5] Kobayashi, S., A theorem on the affine transformation group of a Riemannian manifold, Nagoya Math. J. 9 (1955), 39-41.

[6] Kobayashi, S., Principal fiber bundles with the 1-dimensinal toroidal group, to appear in Tohoku Math. J.

[ 7 ] Lichnerowicz, A., Espaces homogènes kählériens, Colloque de Géométrie Différentielle, Strasbourg (1953), 171-184.

[8] Lichnerowicz, A., Sur les groupes d'automorphismes de certaines variétés kählériennes, C. R. Acad. Sci. Paris, 239 (1954), 1344-1345.

[ 9 ] Nomizu, K., Studies on Riemannian homogeneous spaces, Nagoya Math. J. 9 (1955), 43-56.

[10] de Rham, G., Sur la réductibilité d'un espace de Riemann, Comm. Math. Helv. 26 (1952), 328-344.

[11] Schouten, J. A. and Yano, K., On pseudo-Kählerian spaces admitting a continuous group of motions, Indagationes Math. 17 (1955), 565-570.

\section{University of Washington}

Nagoya University 